\title{
Simulation in Wood Science: using the Process Gluing as an Example
}

\author{
Carina Rößler ${ }^{1,2 *}$, Martin Riegler ${ }^{1}$ \\ ${ }^{1}$ Wood K plus, Competence Centre for Wood Composites and Wood Chemistry, Area Wood Materials \\ Technologies, Konrad-Lorenz-Strasse 24, 3430 Tulln, Austria; *carina.poell@asak.at \\ 2 Institute of Analysis and Scientific Computing, TU Wien, Wiedner Hauptstrasse 8-10, \\ 1040 Vienna, Austria
}

SNE 30(2), 2020, 67 - 72, DOI: 10.11128/sne.30.tn.10514

Received: July 20, 2019 (Selected ASIM SST 2018 Postconf. Publ.), Accepted: December 15, 2019

SNE - Simulation Notes Europe, ARGESIM Publisher Vienna,

ISSN Print 2305-9974, Online 2306-0271, www.sne-journal.org

\begin{abstract}
In this paper, a mathematical model for the sub-process of gluing in the manufacturing process of particleboards is presented. The model describes the application of resin droplets onto wood particles on laboratory scale. At the moment the model and simulations are carried out for the problem considered in two dimensions. The developed mathematical model uses lattice gas cellular automata for the movement of the wood particles, and random walk for the movement of the resin droplets. Feasibility was shown with simulation results of a greatly simplified mathematical model in two dimensions. This model was developed further for a better fitting to the real process. First simulation results of this more detailed model are presented. The next step will be the specification of how validation will be performed. In future, it is planned to create a mathematical model in three dimensions.
\end{abstract}

\section{Introduction}

The industrial manufacturing process of wood-based composites consists of several consecutive subprocesses. The quality of the final product depends on the raw material and the different processing steps. To ensure a high quality of the product, while optimizing the use of energy and resources, adjustable process parameters need to be continuously adapted [1]. Simulations of potential measures can predict the impact of these measures and hence avoid possible costs of rejects.
This generates a deeper understanding of the manufacturing process and enables a more efficient usage of resources.

Based on a value benefit analysis, the sub-process gluing was selected for modelling and simulation.

The wood particles are located in a mixer and thus are moved. Due to the position in the mixer the particles have a local boundary condition. Their movement is locally limited (chips are located at the bottom of the mixer), unless they are transported upwards from the mixer. The resin is sprayed from above into the mixer using nozzles. The resin drops hit the moving wood particles. The aim is to describe the movement and interactions of the wood and resin particles, which results in computing the resin distribution. Appropriate geometries for resin particles and wood particles needed to be selected and different size distributions to be applied. The rules for the different kinds of collisions needed to be defined. In the case of a collision between wood and resin, a statistical distribution was used for calculating the quantity of resin, which penetrates, adheres and passes to another wood particle (lubrication effect).

\section{Gluing}

In this section, the process of gluing is described according to [2], [3], [4] and [5]. Gluing is a sub-process of the manufacturing process of particleboards. In this process step the wood particles are glued with the resin formulation, which is sprayed into the mixer using nozzles. Based on [2] the typical dimensions of the wood chips are: length $8-10 \mathrm{~mm}$, width $1.5-3.5 \mathrm{~mm}$ and thickness $0.25-0.4 \mathrm{~mm}$. In [3] it is stated that the diameter of the resin droplets is between $30 \mu \mathrm{m}$ and $100 \mu \mathrm{m}$. 
The goal of gluing is to equally distribute the resin droplets on the surface of the wood particles, which is important for the bonding of the wood particles. This has a strong impact on the properties of the final particleboard. In the following, the process of gluing is considered on laboratory scale by using a so-called ploughshare mixer, which is shown in Figure 1.

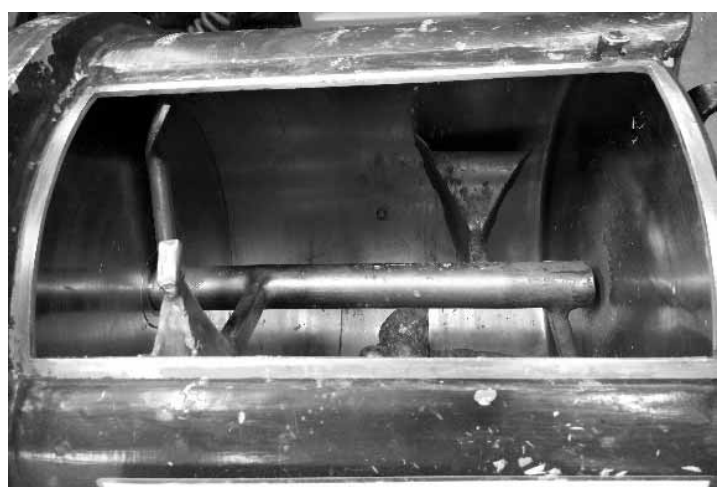

Figure 1: Picture of a ploughshare mixer.

Within the mixer used on laboratory scale, there are four ploughshares (also called mixing arms), a milling head, and an opening for the nozzle. First, the wood chips are located at the bottom of the mixer. Due to the mixing arms the wood particles are moved within the mixer, which constitutes a local boundary condition. The resin is sprayed into the mixer using a nozzle. The resin droplets hit the moving wood particles and thus the wood particles are glued.

\section{Model}

At the moment the model is developed for the two dimensional problem. For modelling the movement of the wood chips a lattice gas cellular automaton (LGCA) is used. The common LGCA is described in [6]. For using LGCA in this setting, the method has to be extended and modified, which will be explained later. For the LGCA a lattice, states and the evolution $\varepsilon$, where $C$ is the collision operator and $S$ is the streaming operator,

$$
\varepsilon=S \circ C
$$

have to be defined. The movement of the resin droplets is realised by a random walk across the lattice used in the LGCA.

The elements, which are used within this model, and the properties of the environment are described in the following.
- wood particles: The shape of the wood particles is considered to be a rectangle.

- resin particles: The shape of the resin droplets is considered to be a circle.

- resinating mixer: The geometry of the cross-sectional area of the resinating mixer is assumed to be a circle.

- ploughshares/mixing arms: The ploughshares move along a circle line.

- time interval

- grid width

In the following, the main elements of the LGCA are considered in more detail. In [7], which is a previous publication of the model, these are already described.

\subsection{Lattice}

The lattice is built by nodes, which are the vertices of equilateral triangles. Therefore, the lattice shows hexagonal symmetry. For obtaining "smooth" objects (circle, which builds the resinating mixer; rectangles, which build the wood particles; circles, which build the resin droplets), a suitable grid width has to be chosen.

In Figure 2 above, the black points are the vertices of the equilateral triangles, which are the nodes of the lattice. The red dashed line represents the wall of the resinating mixer. In Figure 2 below, a small part of the cross-sectional area of the mixer is depicted. The black lines are the edges of the equilateral triangels and the smaller colored triangles represent the directions of movement.

According to [6] at each node there are six cells, which represent the six directions of movement. These correspond to the side lengths of the equilateral triangles and are the so-called lattice velocities. These common lattice velocities are given in equation (2) for $i=$ $1, \ldots, 6$.

$$
c_{i}=\left(\cos \left(\frac{\pi}{3} i+\frac{\pi}{2}\right), \sin \left(\frac{\pi}{3} i+\frac{\pi}{2}\right)\right)
$$

On the one hand, wood particles are stationary, if they are not moved by a mixing arm. On the other hand, resin particles can be stationary, if they stick to the wall of the resonating mixer. Due to the fact that particles can have velocity zero, the common LGCA has to be extended regarding the possible lattice velocities. Therefore, at each node a seventh cell is added, whereby its lattice velocity is zero. 
Thus, the lattice velocity of this cell is defined by equation (3).

$$
c_{0}=(0,0)
$$

Due to the different scale of the wood and resin particle, and the corresponding grid width, the wood particles extend over several nodes of the lattice, which is not provided within the common LGCA. Therefore, some specifications regarding the states, collisions and streaming are necessary.

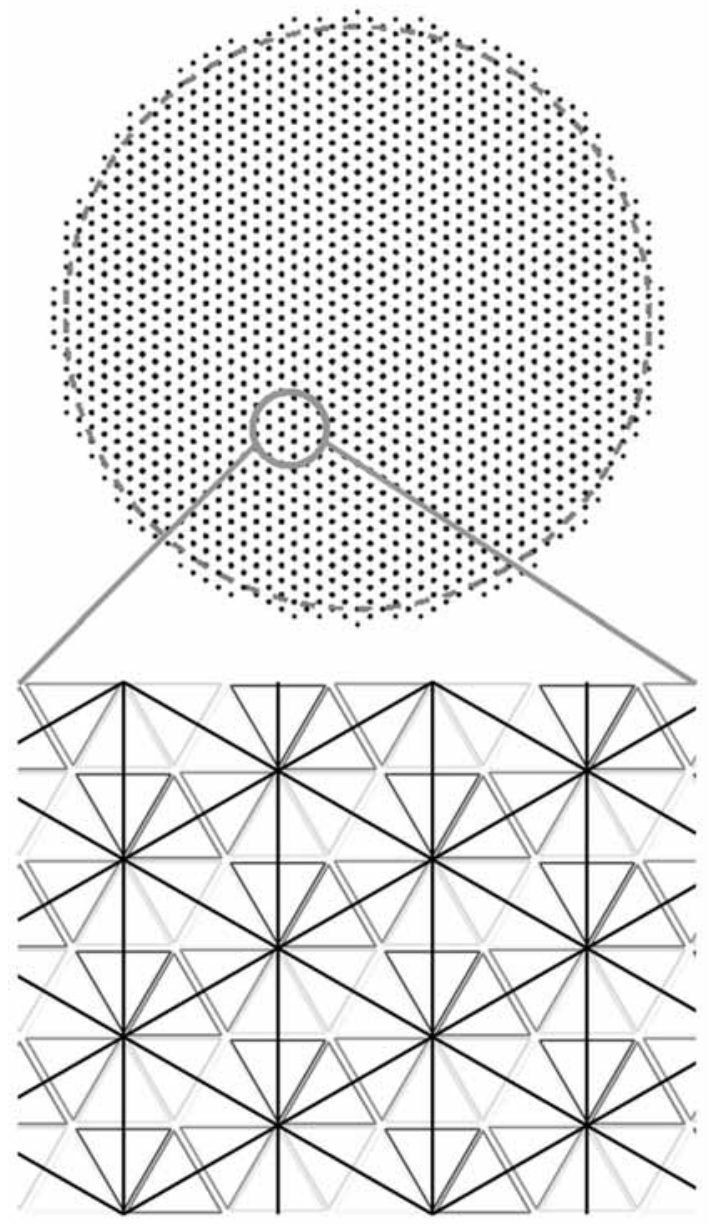

Figure 2: Schematic depiction of the lattice points and the lattice velocities.

\subsection{State}

Each cell of a node must have a so-called state. The state indicates what kind of particle or part of the resinating mixer is located at a certain position. There are certain states, which are described in the following.
- empty: The state "empty" is used for the air, which will not be modelled, and for the definition of the state of a node, which will be explained later.

- wall: The state "wall" is used for the rigid body of the ploughshare mixer.

- ploughshare/mixing arm: For the moving mixing arms this state is used.

- milling head: The state used for the milling head.

- wood: The state for parts of a wood particle without resin.

- resin: The state for the resin droplets.

- glued wood: The state for parts of a wood particle, where resin is adhered, i.e. the state wood is changed to glued wood, if a wood particle and a resin particle collide with each other. After such a collision the resin droplet penetrates into the wood particle over time. Furthermore, if a glued wood particle collides with an unglued wood particle, part of the resin droplet passes to the unglued wood particle, which is called lubrication effect. In section 2.3 these behaviors will be included in the collision rules.

Corresponding to the dimensions of the wood particle and the grid width, the wood particle expands over several nodes. For each node, which is occupied by the wood particle, the state of the cell, which corresponds to the direction of movement, is set to wood. Due to the fact, that each node, which is occupied by the same wood particle, has to move in the same direction. For the other cells of the nodes, the states are set to empty. According to the described situation the cells, which are set empty, of a node, which belongs to a wood particle, are also occupied by the wood particle. Thus, a modification of the common LGCA is necessary. Therefore, the state of a node is introduced. It is defined as wood, if one cell at a node has state wood or glued wood. In Figure 3 an example for this approach is shown schematically. The wood particle occupies three nodes of the lattice, which is indicated by the brown hexagons. The underlying lattice is depicted by the black lines. The brown circles represent the cells with state wood. These cells are chosen corresponding to the direction of movement, which is a certain lattice velocity, of the wood particle. The states of the other cells have to be empty due to the occupation by the wood particle. 


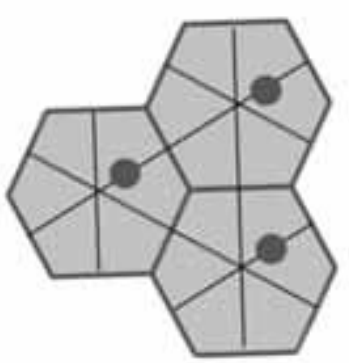

Figure 3: Exemplary illustration of a wood particle, which occupies three nodes.

\subsection{Collision}

As described in section 2.2 several kinds of states are used. Therefore, the rules for all possible types of collisions have to be defined. In the following, these are illustrated using collisions, where two particles or a particle and a machining part are involved. Of course, it is possible that collision of more than two particles can occur.

First, the collision rule for resin particles is defined. For this collision a perfectly inelastic collision is considered, thus after collision there is one bigger resin particle. In Figure 4 the collision of two resin particles is depicted schematically.

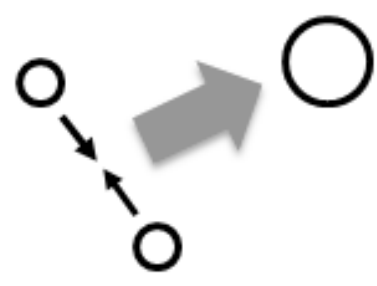

Figure 4: Schematic illustration of the collision of two resin particles.

Next, the collision rule for wood particles is defined. An elastic collision is used for this case, which is shown in Figure 5.
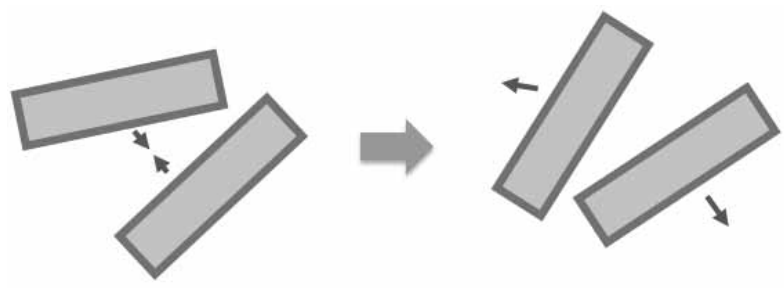

Figure 5: Schematic illustration of the collision of two wood particles.
Due to the porous structure of wood, it can absorb liquids. This is an important fact, which has to be included in the collision rule for wood and resin particles. If such a collision takes place, the resin particle sticks onto the wood particle. Furthermore, a part of the resin particle is absorbed by the wood particle, which is called penetration. In Figure 6 the collision of a wood and resin particle and the penetration are depicted schematically.

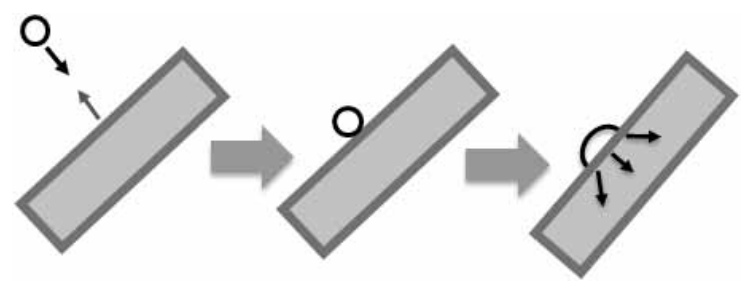

Figure 6: Schematic illustration of the collision of a wood particle and a resin particle.

Next, the collision of a glued wood and an unglued wood particle is considered. This type of collision is modelled as an elastic collision. Furthermore, in this case a part of the resin particle on the surface of the glued wood particle passes to the other wood particle, which is called lubrication effect. In Figure 7 this behaviour is shown schematically. For the collision of two glued wood particles a similar behaviour occurs, i.e. part of the resin drops can pass from one wood particle the other one.

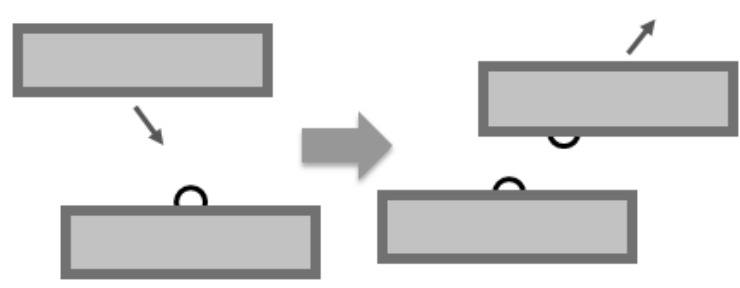

Figure 7: Schematic illustration of the collision of a glued wood and an unglued wood particle.

For all types of collisions mentioned above the velocity of the particles after collision is chosen randomly based on the lattice velocities. This assumption is used for simplification of the implementation.

In the following the collision of a wood particle with parts of the resinating mixer are considered. If a wood particle collides with the wall of the resonating mixer or a mixing arm, an elastic collision takes place. In Figure 8 this type of collision is shown by means of the wall. 

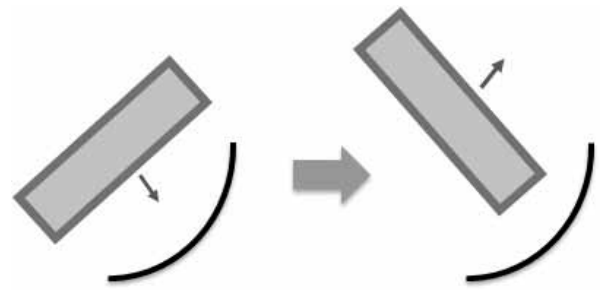

Figure 8: Schematic illustration of the collision of a wood particle and the wall of the resinating mixer.

In contrast to the collision described above, an inelastic collision is used as model for a collision of a resin particle with parts of the mixer. In case of a collision with the wall, the resin particle sticks onto the wall. Therefore, the velocity of the resin particle after collision is zero. In Figure 9 this type of collision is shown. If a resin drop collides with a mixing arm, the velocity of the resin droplet after collision corresponds to the velocity of the mixing arm.
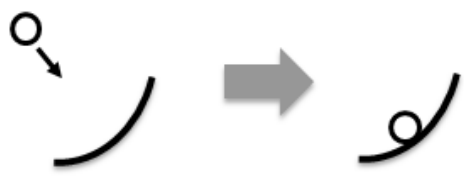

Figure 9: Schematic illustration of the collision of a resin particle with the wall of the resinating mixer.

\subsection{Streaming}

For the streaming of the wood and resin particles, different approaches are used.

Wood particles. If the ploughshares do not move or do not touch a certain wood particle, the wood particle is stationary. Therefore, in the streaming step the wood particle does not move.

If the velocity is not equal to zero, the cells with state wood move according to the corresponding lattice velocity. Due to the technique described in section 2.2 it is guaranteed, that each node of a specific wood particle moves across the same lattice velocity.

Resin particles. The streaming of the resin particles is modelled by a random walk. Due to gravitational acceleration the resin droplets cannot move upwards. Therefore, not all lattice velocities are allowed for the movement of the particles. A random variable is used for choosing one of the possible lattice velocities. After determination of a permitted lattice velocity, the resin particle moves according to this choice across the lattice.
For a resin particle, which sticks onto the wall, the velocity is zero. If it adheres to a mixing arm, the velocity is set to the velocity of the mixing arm.

\section{Simulation}

For simplification of the implementation for the two dimensional model, some assumptions were made:

- The milling head and mixing arms are not realized.

- All wood particles have the same dimensions.

- All wood particles, which are not stationary, have the same speed.

- All resin droplets have the same size and velocity until they adhere on a wood particle or a part of the resonating mixer.

- The velocity after collision of the wood particles is determined randomly.

- The penetration of resin into the wood particle is not implemented.

As a further remark, it has to be mentioned, that not all parameters needed for the simulation can be measured by the real system. Therefore, not all parameters correspond to the real system, which has to be included in the interpretation of the simulation results.

The model was implemented using MATLAB R2015b. In Figure 10 a snapshot of a simulation run is shown, whereas the wood particles are shown in brown and the resin droplets in blue.

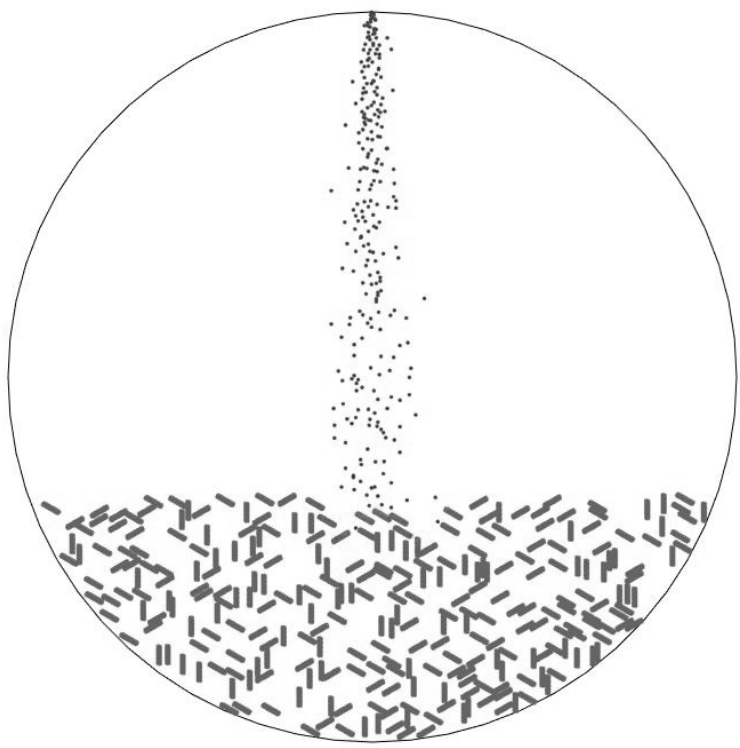

Figure 10: Movement of wood particles (brown) and resin droplets (blue) during simulation.

SNE 30(2) - 6/2020 71 
As illustrated in Figure 10, the resin droplets are moving downwards from the top. Areas of wood particles, which are glued, i.e. a collision of a resin droplet or a glued wood particle and an unglued wood particle took place, are shown in red. For obtaining quantifiable results, the relative frequency of glued wood chips in relation to the total number of wood chips is calculated. First results of this computation are shown in [7].

\section{Plans for Validation}

It is planned to validate the results using experiments on laboratory scale, since validation is important for the connection between the model and the reality. Regarding the behaviour of wood particles and resin droplets after collision, contact angle measurements will be performed for determining the time dependent behaviour (height, spreading) of the droplet on the wooden surface. In Figure 11 a schematic illustration of the technique for measuring the contact angle $\theta$ is shown.

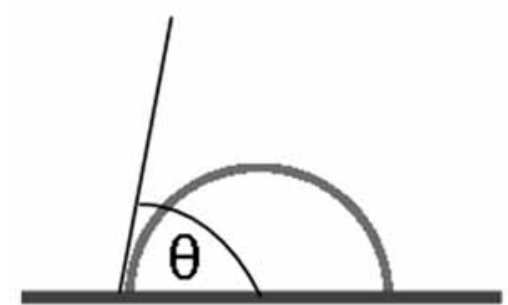

Figure 11: Schematic depiction of contact angle measurement.

Furthermore, for the penetration images of a tiny part of a wood particle, which is glued, can be analysed under a microscope. The lubrication effect will be validated by gluing a wooden board and check the weight before and after it got in contact with an unglued wooden board.

The final result of the sub-process gluing can hardly be measured by a defined criterion, therefore the validation of the final simulation results is limited.

\section{Conclusion and Outlook}

First simulations showed feasibility of the developed mathematical model. The next steps include improving the mathematical model and simulations in two dimensions. Furthermore, the validation of the simulation results will be carried out. Future plans include creating a mathematical model for the process in three dimensions.

\section{References}

[1] Riegler M, Spangl B, Weigl M, Wimmer R, Müller U. "Simulation of a real-time process adaptation in the manufacture of high-density fibreboards using multivariate regression analysis and feedforward control". Wood Science and Technology, vol. 47, pp. 1243-1259, 2013.

[2] Wagenführ A, Scholz F. Taschenbuch der Holztechnik. Carl Hanser Verlag GmbH Co KG. 2012.

[3] Rowell RM. Handbook of wood chemistry and wood composites. CRC press. 2005.

[4] Deppe H, Ernst K. Taschenbuch der Spanplattentechnik. DRW, Leinfelden-Echterdingen, 4th edition. 2000.

[5] Dunky M, Niemz P. Holzwerkstoffe und Leime: Technologie und Einflussfaktoren. SpringerVerlag. 2002.

[6] Wolf-Gladrow DA. Lattice-gas cellular automata and lattice Boltzmann models: an introduction. Springer. 2004.

[7] Rößler C, Riegler M, Breitenecker F. Modeling and simulation of moving wood chips and resin droplets within a resinating mixer using lattice gas cellular automata. IFAC-PapersOnLine, Volume 51, Issue 2, 2018, Pages 55-60.

Acknowledgements. The authors gratefully acknowledge the support from the Austrian Research Promotion Agency for funding as well as the Technische Universität Wien and the University of Natural Resources and Life Sciences for providing infrastructure. 